\title{
Effets des demi-lunes associées au scarifiage sur les productions fourragères en région sahélienne du Burkina Faso
}

\author{
André KIEMA $^{1^{*}}$, Aimé Joseph NIANOGO ${ }^{2,3}$, Chantal Yvette KABORE-ZOUNGRANA ${ }^{2}$ \\ et Blama JALLOH ${ }^{2}$ \\ ${ }^{I}$ Institut de l'Environnement et de Recherches Agricoles; DRREA / Sahel-Dori, BP : 80. Province du Séno, \\ Burkina Faso. \\ ${ }^{2}$ Université Polytechnique de Bobo-Dioulasso, Institut du Développement Rural (IDR), B.P. 1091, \\ Burkina Faso. \\ ${ }^{3}$ Union Mondiale pour la Conservation de la Nature (UICN), 01 BP. 3133 Ouagadougou 01, Burkina Faso. \\ *Auteur correspondant, E-mail: andre_kiema@yahoo.fr; \\ Tél. : (226) 404600 54, Tel: (00226) 70113038.
}

\section{RESUME}

L'effet des demi-lunes associées au scarifiage sur la production fourragère a été étudié dans quatre terroirs sahéliens. Des parcelles d'observation de un ha sur terrain aménagé en demi-lune et un ha sur un témoin respectif ont été mises en place. Les observations ont concerné l'analyse de la composition floristique, la valeur fourragère et le recouvrement. Les résultats obtenus montrent une amélioration de la composition floristique et le recouvrement de la végétation. Les espèces fourragères telles Panicum laetum (+9,8\%), Cassia obtusifolia $(+17,1 \%)$ et Alysicarpus ovalifolius $(+2,7 \%)$ ont connu une amélioration tandis que Schoenefeldia gracilis $(-30 \%)$ et Eragrostis tenella $(-1,1 \%)$ ont subit une régression. La production de fourrage a augmenté significativement $(\mathrm{P}<0,05)$ entre les parcelles aménagées $\left(2115,9 \mathrm{~kg}\right.$ de $\mathrm{MS}$.ha $\left.{ }^{-1}\right)$ et les témoins $(463 \mathrm{~kg}$ de MS.ha $\left.{ }^{-1}\right)$. Cependant, l'apparition de Cassia obtusifolia en très forte contribution spécifique suggère que des essais d'alimentation soient menés sur cette espèce pour mieux valoriser l'impact des aménagements en demilune surtout qu'elle est bien appétée à l'état sec. Les demi-lunes + scarification sont rentables mais des recherches doivent cependant être menées pour trouver les modalités de gestion des espaces aménagés, afin d'éviter leur surexploitation et leur dégradation.

(C) 2012 International Formulae Group. All rights reserved.

Mots clés : Pâturage, fourrage, dégradation, Sahel, Burkina Faso.

\section{INTRODUCTION}

Au Burkina Faso, le processus de désertification se caractérise par une forte dégradation des terres et du couvert végétal principalement en raison des variations climatiques et des activités humaines.

L'aggravation du phénomène par l'érosion hydrique et éolienne fait qu'aujourd'hui plus de $30 \%$ des superficies exploitables du pays sont considérés impropres à la production parce que dégradés (De Wispelaere, 1990). Cela constitue un phénomène écologique et une contrainte majeure à l'utilisation durable de certaines ressources naturelles. 
Les conséquences de cette érosion se manifestent directement sur la végétation. C'est ainsi que la dynamique des peuplements herbacés et ligneux se caractérise par deux situations extrêmes que sont la régression des espèces végétales sélectives et l'expansion des espèces rustiques. Les végétaux ligneux font l'objet d'une intense exploitation (Toutain et De Wispelaere, 1978). Ils sont détruits lors des défriches ou utilisés à des fins diverses : pâture et exploitation des bois d'œuvre et de service (Claude et al., 1991).

Face à cette situation, de nombreuses actions de gestion des ressources naturelles ont été initiées et sont appuyées par des partenaires au développement dans le but d'améliorer et de restaurer les ressources naturelles (sol et végétation). C'est dans ce cadre que s'inscrit cette étude dont l'objectif est d'évaluer l'impact de la restauration des pâturages de glacis par des demi-lunes associées au scarifiage. Cette technique est nouvellement expérimentée par les producteurs avec l'appui des structures de développement rural dans la récupération des terres dégradées de glacis.

\section{MATERIEL ET METHODES \\ Sites d'étude}

L'étude s'est déroulée dans le Sahel burkinabè compris entre le $13^{\text {ème }}$ degré et le $15^{\text {ème }}$ degré de latitude Nord (Claude et al., 1991). Quatre sites d'étude localisés dans les villages de Gnagassi, Koréa, Kryollo et Ekéou ont fait l'objet d'évaluation. Les activités socio-économiques dominantes de ces villages sont l'agriculture et l'élevage. L'élevage constitue pour ces derniers une des principales composantes économiques et des plus rémunératrices du secteur primaire. Il est de type extensif avec des modes de conduite du bétail dominés par les pratiques d'exploitation traditionnelle des ressources pastorales (pâturages naturels, champs, eau, etc.). Cette économie est donc essentiellement basée sur la valorisation du capital terre et/ou ressources naturelles.

\section{Dispositifs} échantillonnage

expérimentaux

et

Les travaux d'aménagement ont été effectués sur les terres de pâturage des villages sélectionnés à raison de leur représentativité pour la région du Sahel. Sur chaque pâturage, les aménagements ont été effectués selon les techniques de demi-lune (DL) et de scarifiage (SC). La DL est une technique de récupération des sols dégradés par économie de l'eau de surface. Elle s'applique aux sols dégradés en surface c'està-dire les terres de plateau et les glacis à pente inférieure à $3 \%$. Elle consiste à creuser des cuvettes de la forme de demi-cercles, les terres de déblais étant disposées en bourrelets semi-circulaires. Pour cette étude, les aménagements sous forme de demi-lune ont été réalisés à la charrue delphino. Les DL sont longues de 5 à $6 \mathrm{~m}$ et larges de 1 à $1,5 \mathrm{~m}$. Elles sont installées de façon discontinue sur des lignes parallèles, espacées de 2,5 à $3 \mathrm{~m}$ et disposées perpendiculairement à la pente du glacis aménagé. Entre les lignes de DL, un SC (sur les espaces de 2,5 à $3 \mathrm{~m}$ ) a été fait pour renforcer l'effet sur la restauration de la végétation.

Le SC est une pratique qui consiste à travailler le sol par grattage de la couche superficielle avec un tracteur attelé à un instrument à dents, en vue d'ameublir les 10 premiers $\mathrm{cm} \mathrm{du}$ sol. Les travaux de grattage ont été effectués intégralement entre les espaces des lignes de demi-lunes.

Les travaux de DL et de SC ont tous été effectués à sec. Par la technique à sec, on obtient un travail très irrégulier du sol. Ces deux pratiques sont des techniques culturales utilisées pour lutter contre l'encroûtement des sols par son ameublissement. Elles permettent d'optimiser l'utilisation de l'eau de pluie à la parcelle (Photo 1).

En moyenne, 392 DL ont été réalisées par ha sur les superficies aménagées pour une superficie de $2695 \mathrm{~m}^{2}$. Cela représente $26,95 \%$ de surface couverte par les DL et $73,05 \%$ pour le SC. Tous les aménagements ont été réalisés sur des glacis plus ou moins 
dégradés avec des superficies totales variant entre 30 et 60 ha. Sur chaque site d'étude, des observations (mesures) sur terrain aménagé (en DL et SC) et sur terrain témoin (sans travail du sol) ont été effectuées sur des parcelles représentatives de ha. Quatre parcelles témoins ont été identifiées sur les mêmes unités de végétation en zone non aménagée située en continuité de celles aménagées et représentatives des conditions naturelles (Figure 1).

\section{Composition floristique}

Les inventaires de la végétation herbacée ont été réalisés à l'aide de la méthode des «points quadrats alignés» (Daget et Poissonet, 1971). La méthode consiste à tendre un double décamètre audessus du tapis herbacé et à effectuer une lecture verticale tous les $20 \mathrm{~cm}$ à l'aide d'une tige métallique à bord effilé. Tous les contacts avec les feuilles ou les chaumes sont pris en compte. Par convention, une espèce ne doit être notée qu'une fois par point de lecture. Chaque ligne ainsi matérialisée permet de faire 100 observations.

Pour chaque site d'étude, cinq lignes de points quadrats par traitement de DL, de SC et de témoin ont été réparties au hasard, ce qui correspond à un nombre suffisant déterminé par l'intervalle de confiance (IC) calculé au fur et à mesure sur le terrain. L'IC est calculé à partir de l'effectif cumulé ligne par ligne des contacts de l'espèce dominante sur l'effectif cumulé des contacts enregistrés par l'ensemble des espèces (Boudet, 1991).

$I C= \pm 2 \sqrt{\frac{n(N-n)}{N^{3}}}$

«N» est l'effectif cumulé des contacts de l'ensemble des espèces,

«n»l'effectif cumulé des contacts de l'espèce dominante.

Ces observations ont permis de calculer :

- la fréquence spécifique (FS) correspondant à la proportion des espèces au sol.
- la fréquence centésimale (FC) est égale au rapport en (\%) de la FS sur le nombre (N) de points échantillons.

$$
F C=\frac{F S}{N} * 100
$$

Quand le nombre de points est très élevé, la FC, représente le recouvrement (Godron et al., 1968).

- La contribution spécifique (CSi) est définie comme le rapport de la FS de cette espèce à la somme des FS de toutes les espèces recensées.

$C S_{i}=\frac{F S_{i}}{\sum_{i=1}^{n} F S_{i}} * 100$

Avec $\mathrm{n}=$ le nombre des espèces.

$\mathrm{CS}_{\mathrm{i}}$ et $\mathrm{FS}_{\mathrm{i}}$ sont les contributions et fréquences de l'espèce $\mathrm{i}$ et $\mathrm{n}$ le nombre d'espèces. CSi est la fréquence relative de l'espèce i dans l'ensemble des fréquences spécifiques observées.

\section{Estimation de la valeur pastorale}

La valeur relative des espèces encore appelée indice de qualité spécifique (IS) a été établie à partir d'une échelle de cotation de 0 à 5 à l'issue de la synthèse de nombreux travaux (Gaston et Botte, 1971; Kiema, 1994 ; Kaboré-Zoungrana, 1995). Ainsi, les espèces fourragères ont été classées en excellentes (indice est égal à 5), très bonnes (indice égal à 4), bonnes (indice égal à 3), moyennes à médiocres (indice égal à 2), médiocres à mauvaises (indice égal à 1), espèces à valeurs nulles (indice égal à 0 ).

Ces indices permettent d'effectuer des comparaisons très instructives entre les unités de végétation d'une même région (Daget et Poissonet, 1972). La valeur pastorale (VP) a été déduite en appliquant la formule proposée par Daget et Poissonet (1971).

$V P=0,2 \sum C S_{i} * I S_{i}$

où 0,2 est un coefficient qui permet d'exprimer VP en $\%$.

\section{Evaluation de la biomasse}

La méthode utilisée est celle de la récolte intégrale à partir des carrés de $1 \mathrm{~m}^{2}$ (Levang, 1978). Dans chaque site et pour 
chaque traitement de DL, de SC et de témoin, 12 carrés ont été mesurés. Les échantillons fauchés ont été séparés suivant les espèces dominantes et pesés frais. Un échantillon de $500 \mathrm{~g}$ frais de chaque groupe de végétaux a été prélevé par parcelle et mis à l'étuve à 105 ${ }^{\circ} \mathrm{C}$ pour la détermination de la matière sèche.

\section{L'évaluation des coûts de production}

Les coûts de production du fourrage sur les parcelles ont été évalués en tenant compte des charges d'aménagement de demi-lunes et scarifiage qui sont respectivement de 40000 et 20000 FCFA/ha (PSB, 2003) ; des rendements de fourrage exploitable (Grouzis, 1988; Bougouma et al., 2001), des charges d'exploitation (main d'œuvre pour la fauche) et de transport. La durée d'amortissement considérée selon l'efficacité des DL et du SC a été estimée respectivement à trois (03) et deux (02) ans. Les marges nettes de production ont été déduit des différentes charges et des prix de vente du fourrage qui ont été évaluées à $60 \mathrm{FCFA} / \mathrm{kg}$ en saison sèche (période optimale de vente du fourrage) (Bougouma et al., 2001).

\section{Evaluation du bilan des nutriments}

Le bilan des éléments nutritifs a été obtenu par les analyses des échantillons de sols et de fourrage. Les fourrages ont été prélevés dans les placeaux de récolte intégrale de la végétation immédiatement lors de l'évaluation de la biomasse. Les sols ont également aussitôt été prélevés dans ces placeaux de $1 \mathrm{~m}^{2}$ sur l'horizon $0-20 \mathrm{~cm}$ considéré comme le siège du développement racinaire maximal de la plupart des herbacées. Les échantillons composites de sol d'un même niveau de gradient ont été stockés ensemble. Au total, 12 échantillons de sol et 20 de phytomasse correspondant aux prélèvements sur les DL, les espaces de SC et les témoins ont été prélevés.

Les analyses ont concerné le $\mathrm{pH}$, le carbone, l'azote et le phosphore total pour les échantillons de sol; l'azote et le phosphore assimilable pour les plantes.

\section{Analyses statistiques}

L'estimation de la dynamique du couvert herbacé a été effectuée à partir des fréquences spécifiques. Les comparaisons statistiques de différence entre les espaces traitées et le témoin se sont appuyées sur les principales espèces végétales en utilisant le test de $X^{2}$ au seuil de probabilité de $5 \%$ (Boudet, 1991).

Les résultats ont été soumis par le logiciel Statistical Package for Social Science (SPSS), à des analyses de variance (ANOVA) à un critère de classification afin de tester l'effet des différents traitements (DL et SC) sur la production de phytomasse et les caractéristiques physico-chimiques des sols des parcelles. Le test de Scheffe (1959) a été utilisé pour identifier les moyennes qui diffèrent significativement au seuil de 5\%.

\section{RESULTATS}

\section{Structure et composition floristique}

Les relevés floristiques dans les différents sites font ressortir 40 espèces herbacées sur les parcelles aménagées contre 32 espèces sur le témoin, soit un écart de 8 espèces. Les graminées dominent largement la strate herbacée. Sur les parcelles aménagées, elles sont dominantes avec 28 espèces pour une contribution spécifique (CSi) de 72,2\%. Les légumineuses y sont moins représentées avec 5 espèces pour 21,4\% de CSi. Dans les parcelles témoins, les graminées constituent également le plus grand nombre d'espèces avec $89,9 \%$ de CSi tandis que les légumineuses ne sont représentées que par 4 espèces pour $1 \%$ de CSi (Tableau 1).

$\mathrm{Du}$ point de vue contribution spécifique, il ressort que les aménagements ont entraîné une modification de la composition floristique. Cette recomposition de la végétation est caractérisée par une progression des espèces hygrophiles et une régression des autres types d'espèces. Il existe une variabilité entre sites d'essai mais on peut retenir que les espèces en nette progression sont constituées essentiellement de Cassia obtusifolia $(+17,1 \%), \quad$ Panicum laetum 
(+9,78\%), Alysicarpus ovalifolius (+2,73\%), Borreria radiata $(+1,49 \%)$ et Eragrostis pilosa $(+2,25 \%)$. Les espèces en régression sont représentées par Schoenefeldia gracilis $(-30 \%)$ et Eragrostis tenella $(-1,1 \%)$.

La dynamique s'est également manifestée en ce qui concerne le recouvrement. Les sols nus se sont en effet réduits sur les parcelles aménagées, en moyenne de $-33 \%$, par rapport aux parcelles témoins.

Le nombre de points contact est plus élevé sur les parcelles aménagées (135 points contact / 100 points de lecture) par rapport au témoin (81 points contact / 100 points de lecture) et indique un plus fort recouvrement de la végétation lié à l'aménagement des parcelles.

D’un constat général, les différences enregistrées entre parcelles aménagées et témoins respectifs démontrent clairement les effets améliorateurs des DL $(\mathrm{p}<0,01)$.

\section{Estimation de la valeur pastorale}

La dynamique observée sur la valeur pastorale montre que les aménagements ont améliorés les proportions des espèces fourragères de très bonne $(+10 \%)$ et de médiocre $(+3,4 \%)$ qualité au détriment des espèces de moyenne qualité $(-12 \%)$, plus présentes sur le témoin. La valeur pastorale brute est significativement améliorée ( $\mathrm{P}<$ $0,05)$ sur les parcelles aménagées $(52,6 \%)$ par rapport aux témoins $(50,4 \%)$ (Tableau 2$)$.

\section{Estimation de la biomasse}

La production de fourrage a été influencée par les traitements de DL. En moyenne, la production des parcelles aménagées $\left(2115,9 \mathrm{~kg}\right.$ de $\left.\mathrm{MS}_{\mathrm{h}} \mathrm{ha}^{-1}\right)$ a été supérieure à celle des parcelles témoins (463 $\mathrm{kg}$ de $\mathrm{MS}^{-h^{-1}}{ }^{-1}$. Cette production est particulièrement élevée sur les DL $(5856,4 \mathrm{~kg}$

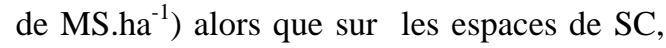
elle a été en moyenne de $873 \mathrm{~kg}$ de ${\mathrm{MS} . \mathrm{ha}^{-1}}^{-1}$ (Figure 1)

La réponse des espèces et des familles d'espèces à l'aménagement est très variable.
Cassia obtusifolia a constitué $74,5 \%$ de la biomasse des parcelles aménagées et $11 \%$ des témoins contre respectivement $23,6 \%$ et $86,3 \%$ pour les graminées. L'analyse de la variance a montré des différences significatives $(\mathrm{P}<0,05)$ pour la production de Cassia obtusifolia et les autres espèces dans les différents traitements alors qu'aucune différence n'a été observée pour les graminées.

Il est à souligner que pour cette espèce, les tiges représentent $49,1 \%$, les feuilles $33,6 \%$ et les gousses $17,3 \%$ des productions. Les parties les plus consommées sont surtout constituées par les gousses durant la saison sèche. Cependant, les différentes proportions varient en fonction du milieu écologique puisque sur les DL, les tiges représentent $50,4 \%$, les feuilles $28,3 \%$ et les gousses $21,3 \%$. Sur les espaces de SC, ces proportions sont respectivement de 47,3\%, 40,9\% et $11,8 \%$. Les espaces de SC produisent plus de feuilles que les sillons de DL (zone humide) qui produisent par contre plus de tiges et de gousses.

\section{Evaluation des coûts de production}

L'évaluation des coûts de production indique que les DL associées au SC sont rentables. Les charges de production ont été étalées en deux ans pour le scarifiage et trois ans pour les demi-lunes. Elles s'élèvent respectivement à 10000 et 13400 FCFA par ha. $\mathrm{an}^{-1}$. Les marges évaluées montrent qu'annuellement le producteur peut produire du fourrage qui rapporte en marges nette 15 150 FCFA.ha ${ }^{-1}$ sur une parcelle aménagée et 7 860 FCFA.ha $^{-1}$ pour le témoin sur la base d'une exploitation sans investissement (Tableau 3).

\section{Bilan des nutriments \\ Caractéristiques physico-chimiques du sol}

Les caractéristiques physico-chimiques des sols aménagés et témoin sont indiquées au Tableau 4. Les teneurs en éléments chimiques des sols sont en général faibles particulièrement pour l'azote et le phosphore. 
Les caractéristiques granulométriques ont été marquées par une amélioration des sols aménagés en sable grossier et fin en raison des phénomènes de sédimentation induits par les travaux de sols. Les autres éléments, limon et argile, sont par contre plus faibles sur les parcelles aménagées. D'un constat général, l'aménagement a eu très peu d'effet sur ces teneurs et l'analyse de variance n'a pas montré de différence significative $(\mathrm{P}>0,05)$ entre les traitements.

Composition chimique des fourrages et production totale d'azote et de phosphore

Les teneurs en matières azotées totales des fourrages (MAT) sont élevées sur les parcelles aménagées en raison certainement d'une fertilité plus élevée mais surtout du fait de l'humidité plus persistante occasionnée par le travail du sol en DL. Cette humidité permet au fourrage de rester vert pendant une période plus longue et d'accroître le temps d'absorption des éléments nutritifs. Contrairement aux teneurs en MAT, celles en phosphore ont été cependant plus élevées sur les parcelles témoins, ceci en rapport avec la teneur de cet élément dans le sol. La légumineuse Cassia obtusifolia récoltée sur les parcelles aménagées a été plus riche en MAT $\left(211 \mathrm{~g}^{\mathrm{kgMS}}\right)$ et en phosphore (756 mg ${ }^{\mathrm{kg} \mathrm{MS}}$ ).

De ce fait, les immobilisations totales d'azote et de phosphore calculées à partir des fourrages produits et de leur teneur en ces éléments montrent que la mobilisation annuelle due à l'effet des traitements de DL a été en moyenne beaucoup plus importante suite aux aménagements. Dans le cas de l'azote, on note les valeurs de $42,4 \mathrm{~kg} \cdot \mathrm{ha}^{-1}$ et 6,3 kg.ha ${ }^{-1}$ respectivement sur la parcelle aménagée et le témoin.

La contribution de l'espèce Cassia obtusifolia apparue à la faveur de l'aménagement est très importante $(35 \mathrm{~kg}$ d'N.ha' ${ }^{-1}$; soit $82,5 \%$ des productions totales) sur la parcelle aménagée. Dans le cas des graminées, le rapport entre parcelle aménagée et parcelle témoin est de 1,39 fois. Les diverses autres espèces contribuent respectivement avec 1,25 et $2,54 \%$ pour l'azote total produit sur les parcelles de DL et des témoins. La productivité sur les DL correspond à 3,17 fois celle de la parcelle aménagée et à 16,02 fois celles des parcelles de SC.

La production totale de phosphore est de 4,29 kg.ha ${ }^{-1}$ sur les parcelles aménagées contre $0,34 \mathrm{~kg} / \mathrm{ha}$ sur le témoin; soit une amélioration de 12,62 fois. La contribution de Cassia obtusifolia $(67,13 \%)$ est également importante sur les parcelles aménagées (Tableau 5).
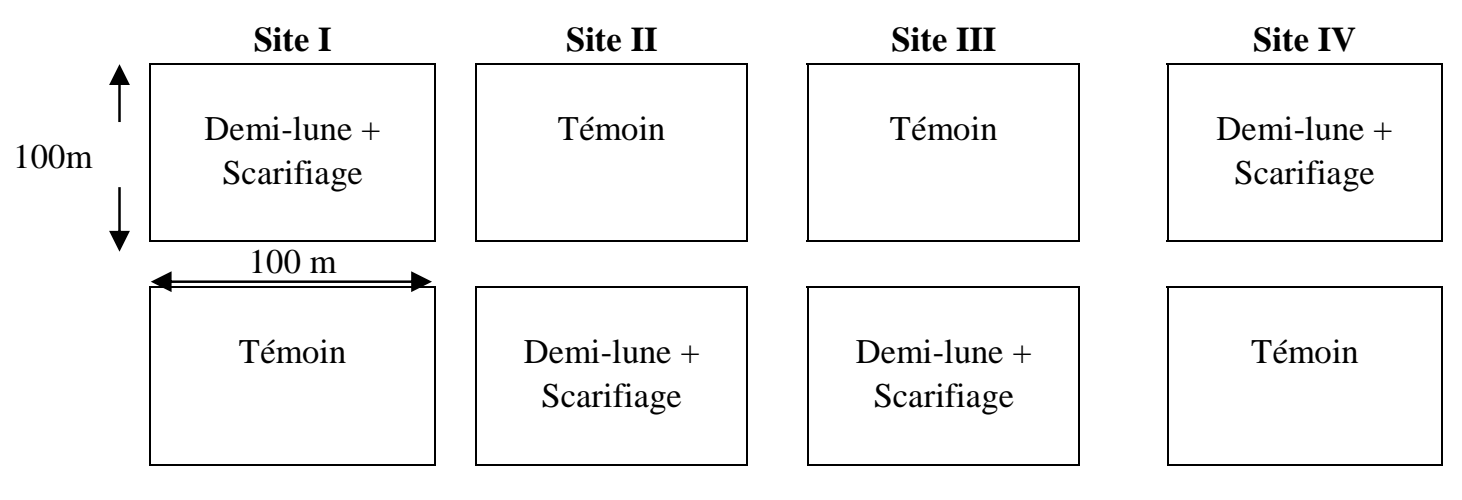

Figure 1: Dispositif expérimental. 
A. KIEMA et al. / Int. J. Biol. Chem. Sci. 6(6): 4018-4030, 2012

Tableau 1: Contributions spécifiques en \% des principales espèces et familles végétales.

\begin{tabular}{|c|c|c|c|c|c|c|c|}
\hline Espèces & Famille des espèces & Demi-lune & $\begin{array}{c}\text { Inter demi-lune } \\
\text { scarifiée }\end{array}$ & $\begin{array}{c}\text { Moyenne } \\
\text { demi-lune + scarifiage }\end{array}$ & Témoin & $\begin{array}{c}\text { Indice de qualité } \\
\text { des espèces }\end{array}$ & Appetibilité \\
\hline Alysicarpus ovalifolius (S. et T.) Léo. & Legumineae & 5,39 & 2,07 & 2,96 & 0,23 & 4 & Très appété \\
\hline Aristida adscensionis $\mathrm{L}$. & Gramineae & 1,23 & 0,93 & 1,01 & 0,47 & 2 & Appété \\
\hline Boerhavia repens $\mathrm{L}$. & Nyctaginaceae & 0,31 & 0,61 & 0,53 & 2,34 & 2 & Appété \\
\hline Borreria radiata $\mathrm{DC}$. & Rubiaceae & 3,73 & 1,8 & 2,32 & 0,83 & 3 & Peu appété \\
\hline Brachiaria distichophylla (T.) Stapf & Gramineae & 1,85 & 0,62 & 0,95 & 0 & 3 & Très appété \\
\hline Brachiaria lata (Schumach.) Hubb. & Gramineae & 2,44 & 2,33 & 2,36 & 3,42 & 5 & Très appété \\
\hline Cassia obtusifolia $\mathrm{L}$. & Legumineae & 38,5 & 9,84 & 17,6 & 0,49 & 2 & Peu appété \\
\hline Chloris pilosa Schum. et Thonn. & Gramineae & 1,54 & 1,09 & 1,21 & 1,28 & 3 & Appété \\
\hline Corchorus tridens $\mathrm{L}$. & Tiliaceae & 0,65 & 0,13 & 0,27 & 0,99 & 3 & Appété \\
\hline Cyperus rotundus $\mathrm{L}$. & Cyperaceae & 0,26 & 1,82 & 1,4 & 0,47 & 2 & Appété \\
\hline Dactyloctenium aegyptium (L.) Willd. & Gramineae & 1,1 & 1 & 1,03 & 1,79 & 4 & Très appété \\
\hline Digitaria horizontalis Willd. & Gramineae & 1,49 & 0,85 & 1,02 & 0,23 & 4 & Appété \\
\hline Eragrostis pilosa (L.) P. Beauv. & Gramineae & 3,43 & 2,13 & 2,48 & 0,23 & 3 & Appété \\
\hline Eragrostis tenella Roem. et Sch. & Gramineae & 1,16 & 8,8 & 6,74 & 7,82 & 3 & Appété \\
\hline Indigofera hirsuta $\mathrm{L}$. & Papilionaceae & 0,06 & 0,03 & 0,04 & 1,66 & 2 & Peu appété \\
\hline Indigofera tinctoria $\mathrm{L}$. & Legumineae & 0,75 & 0,93 & 0,88 & 0,32 & 2 & Appété \\
\hline Ipomea coscinosperma $\mathrm{H}$. ex C. & Convolvulaceae & 1,07 & 0 & 0,29 & 0,56 & 2 & Appété \\
\hline Panicum laetum Kunth & Gramineae & 14,2 & 29,8 & 25,6 & 15,8 & 4 & Very appété \\
\hline Schoenefeldia gracilis Kunth & Gramineae & 17 & 31,5 & 27,6 & 57,2 & 3 & Appété \\
\hline Zornia glochidiata Reichb. ex DC. & Legumineae & 0,56 & 0,25 & 0,34 & 0 & 4 & Très appété \\
\hline Sol nu \% & - & 3,75 & 17,7 & 13,9 & 46,6 & - & - \\
\hline IC \% & - & 2,64 & 3,64 & 3,37 & 4,16 & - & - \\
\hline Nombre de contacts /100 points & - & 199 & 112 & 135 & 81,1 & - & - \\
\hline
\end{tabular}


Tableau 2: Indice de qualité (\%) des différentes catégories d'espèces fourragères.

\begin{tabular}{|c|c|c|c|c|c|}
\hline \multirow[b]{2}{*}{ Espèces dominantes } & \multirow[b]{2}{*}{ Catégories } & \multicolumn{4}{|c|}{ Traitements } \\
\hline & & $\begin{array}{l}\text { Demi- } \\
\text { lune }\end{array}$ & $\begin{array}{l}\text { Inter demi- } \\
\text { lune scarifiée }\end{array}$ & $\begin{array}{c}\text { Demi-lune + } \\
\text { scarifiage }\end{array}$ & Témoin \\
\hline Brachiaria lata & Excellente & $2,4^{\mathrm{a}}$ & $2,3^{\mathrm{a}}$ & $2,4^{\mathrm{a}}$ & $3,4^{\mathrm{a}}$ \\
\hline Panicum laetum, Alysicarpus ovalifolius, Zornia glochidiata, Echinochloa colona & Très bonne & $18,3^{\mathrm{a}}$ & $27,2^{\mathrm{a}}$ & $24,8^{\mathrm{a}}$ & $14,5^{\mathrm{a}}$ \\
\hline Digitaria horizontalis, Eragrostis tremula & Bonne & $8,1^{\mathrm{a}}$ & $8,9^{\mathrm{a}}$ & $8,7^{\mathrm{a}}$ & $7,1^{\mathrm{a}}$ \\
\hline Schoenefeldia gracilis, Aristida spp & Moyenne & $8,6^{\mathrm{a}}$ & $14,8^{\mathrm{a}}$ & $13,1^{\mathrm{a}}$ & $25,1^{\mathrm{a}}$ \\
\hline $\begin{array}{l}\text { Cassia obtusifolia, Setaria pumila, microchloa indica, Corchorus tridens, Commelina } \\
\text { benghalensis, Chloris spp }\end{array}$ & Médiocre & $7,8^{\mathrm{a}}$ & $2,1^{\mathrm{ab}}$ & $3,6^{\mathrm{a}}$ & $0,2^{\mathrm{b}}$ \\
\hline
\end{tabular}

Sur la même ligne les chiffres suivis d'une même lettre ne sont pas significativement différents $(\mathrm{P}<0,05)$.

Tableau 3: Evaluation des coûts et de la rentabilité des aménagements en FCFA par ha ${ }^{\text {an }}$.

\begin{tabular}{|c|c|c|}
\hline Paramètres & Moyenne demi-lune + scarifiage & Témoin \\
\hline 1. Coût fixe FCFA & 23400 & 0 \\
\hline Demi-lune (Coût annuel FCFA) & 13400 & 0 \\
\hline Scarifiage (Coût annuel FCFA) & 10000 & 0 \\
\hline 2. Coût variable FCFA & 5750 & 1400 \\
\hline Main d'œuvre fauche FCFA & 5000 & 1050 \\
\hline Transport du fourrage FCFA & 750 & 350 \\
\hline 3. Rendement fourrage $\mathrm{kg} \mathrm{MS} \mathrm{-} \mathrm{ha}$ & 738,3 & 154,3 \\
\hline Coût de production du kg de fourrage FCFA & 39,48 & 9,07 \\
\hline 4. Prix unitaire du fourrage - $^{-M_{\text {de }} \mathrm{S} \text { en saison sèche }}$ & 60 & 60 \\
\hline 5. Marge brute FCFA & 44300 & 9260 \\
\hline 6. Marge nette FCFA & 15150 & 7860 \\
\hline
\end{tabular}


Tableau 4: Caractéristiques physico-chimiques des sols des parcelles aménagées et du témoin.

\begin{tabular}{lcccc}
\hline Paramètres & Demi-lune & $\begin{array}{c}\text { Inter espace } \\
\text { scarifié }\end{array}$ & $\begin{array}{c}\text { Moyenne demi-lune + } \\
\text { scarifiage }\end{array}$ & Témoin \\
\hline Carbonne g ${ }^{-\mathrm{kg}}$ & 3,15 & 2,41 & 2,61 & 2,63 \\
M O \% & 0,72 & 0,55 & 0,6 & 0,6 \\
Azote en g ${ }^{-\mathrm{kg}}$ & 0,38 & 0,34 & 0,35 & 0,36 \\
Phosphore $\mathrm{mg}-{ }^{\mathrm{kg}}$ & 77,3 & 74,3 & 75,1 & 76,9 \\
pHeau & 6,23 & 6,32 & 6,3 & 6,72 \\
pHKCL & 4,96 & 5,04 & 5,02 & 5,28 \\
Argile \% & 21,1 & 20,8 & 20,9 & 26,8 \\
Limon (grossier et fin) \% & 12,7 & 11,2 & 11,6 & 12,9 \\
Sable (grossier et fin) \% & 66,3 & 68 & 67,5 & 60,4 \\
\hline
\end{tabular}

Tableau 5: Composition chimique et immobilisation d'azote et de phosphore par la biomasse des parcelles aménagées et leurs témoins.

\begin{tabular}{|c|c|c|c|c|c|}
\hline Paramètres & Types de fourrage & Demi-lune & $\begin{array}{l}\text { Espace } \\
\text { scarifié }\end{array}$ & $\begin{array}{c}\text { Moyenne } \\
\text { essai }\end{array}$ & Témoin \\
\hline \multirow{3}{*}{ Teneur en MM (\% MS) } & Cassia obtusifolia & 7,8 & \multirow{3}{*}{9,7} & & \multirow{3}{*}{10,1} \\
\hline & Graminées & 11,8 & & & \\
\hline & Autres espèces & 18,5 & & & \\
\hline \multirow{3}{*}{ Teneur en MAT $\left(^{-\mathrm{kg} \mathrm{MS}}\right)$} & Cassia obtusifolia & 211 & \multirow{3}{*}{131} & & \multirow{3}{*}{127} \\
\hline & Graminées & 145 & & & \\
\hline & Autres espèces & 129 & & & \\
\hline \multirow{3}{*}{ Teneur en $\mathrm{P}\left(\mathrm{mg}-{ }^{\mathrm{kg} \mathrm{MS}}\right)$} & Cassia obtusifolia & 756 & \multirow{3}{*}{647} & & \multirow{3}{*}{725} \\
\hline & Graminées & 685 & & & \\
\hline & Autres espèces & 720 & & & \\
\hline \multirow{4}{*}{$\begin{array}{l}\text { Immobilisation de l'azote } \\
\text { en } \mathrm{kg} / \mathrm{ha} / \mathrm{an}\end{array}$} & Cassia obtusifolia & 111 & 6,93 & 35 & 1,08 \\
\hline & Graminées & 7,71 & 6,81 & 7,05 & 5,06 \\
\hline & Autres espèces & 1,11 & 0,31 & 0,53 & 0,16 \\
\hline & Total & 119 & 14,1 & 42,4 & 6,3 \\
\hline \multirow{4}{*}{$\begin{array}{l}\text { Immobilisation du } \\
\text { phosphore en } \mathrm{kg} / \mathrm{ha} / \mathrm{an}\end{array}$} & Cassia obtusifolia & 3,96 & 2,48 & 2,88 & 0,04 \\
\hline & Graminées & 3,64 & 0,34 & 1,23 & 0,29 \\
\hline & Autres espèces & 0,62 & 0,02 & 0,18 & 0,01 \\
\hline & Total & 8,22 & 2,84 & 4,29 & 0,34 \\
\hline
\end{tabular}




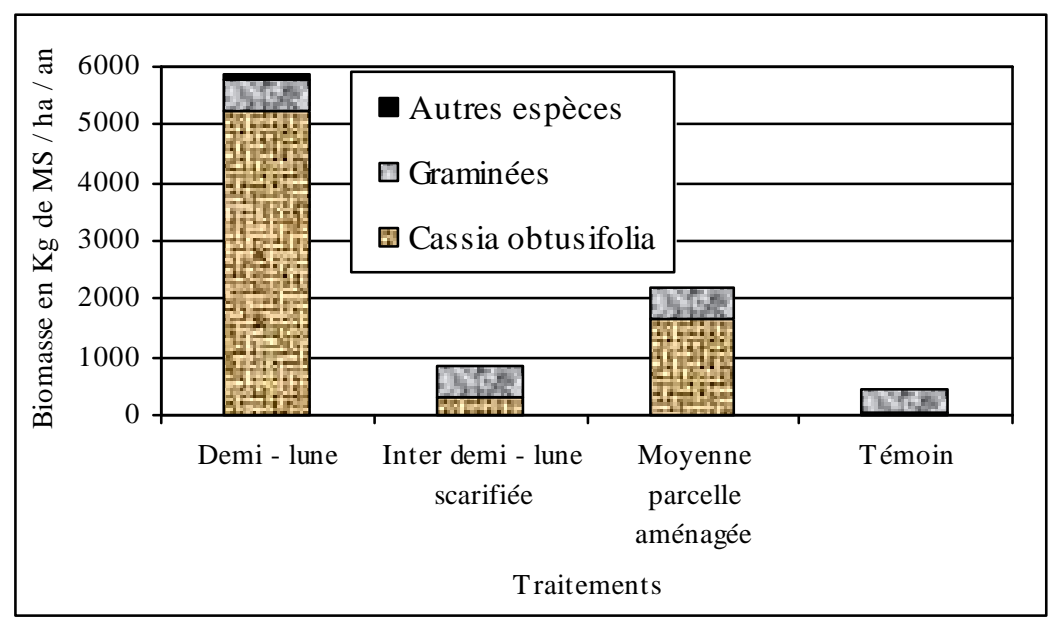

Figure 1: Effets des demi-lunes associées au scarifiage sur la production de phytomasse.

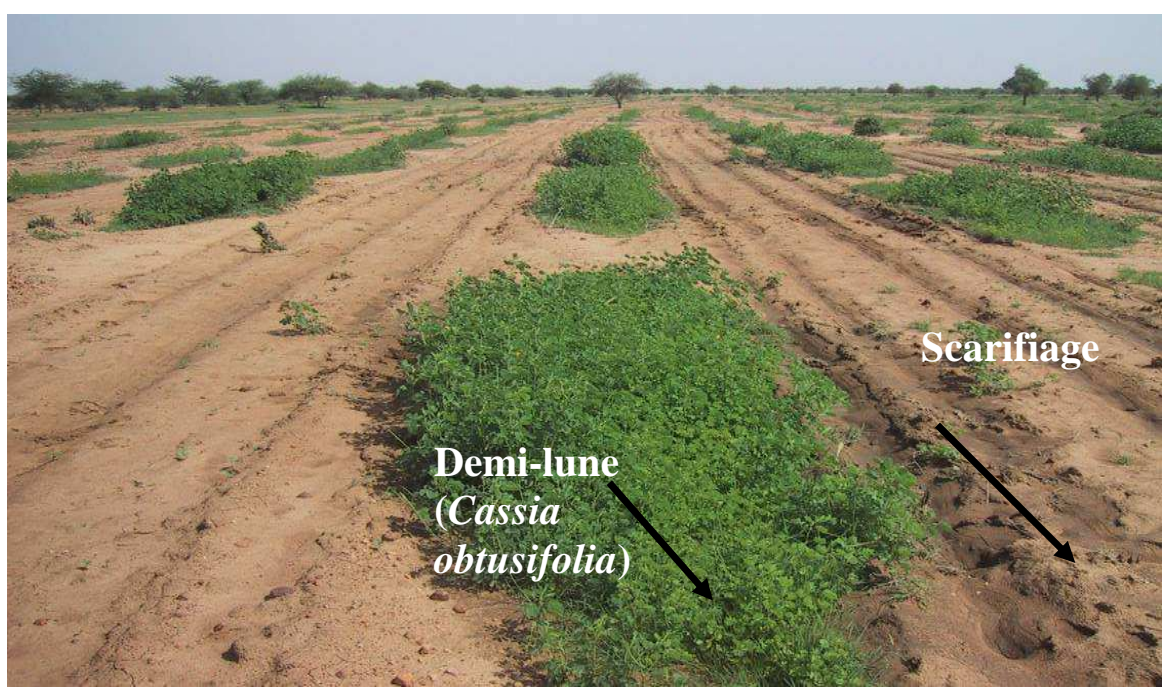

Photo 1 : Demi-lunes associées au scarifiage (en début août).

\section{DISCUSSION}

L'expérience des DL qui est décrite pour la région sahélienne a montré l'intérêt des travaux du sol. L'impact a en effet été évalué en ce qui concerne la structure et la composition de la végétation, la valeur du pâturage, la biomasse produite et les coûts de production.

La composition floristique est un paramètre qui varie en fonction des facteurs écologiques du milieu : gradient de texture, d'humidité, les variations de l'état de surface du sol. Dans les différents sites d'étude, l'impact des demi-lunes sur la composition floristique s'est manifesté par un enrichissement des espèces de 1,3 fois par rapport aux parcelles non aménagées. Cet enrichissement s'est caractérisé par une régression des graminées dont les espèces les plus affectées ont été Schoenefeldia gracilis et Eragrostis spp, et confirment les observations similaires rapportées par (Grouzis, 1988; Kiema et al., 2001). Par contre, les légumineuses ont connu une augmentation 
avec spécifiquement Cassia obtusifolia. Cette espèce peu appétée à l'état frais en saison des pluies a les gousses et les feuilles qui sont plutôt bien consommées en saison sèche.

Le recouvrement global de la végétation a été fortement amélioré car le pourcentage de sol nu a diminué de $-33 \%$ en moyenne.

Les inventaires floristiques ont permis d'évaluer l'ensemble de la flore, les proportions des différentes familles influencées par les traitements effectués sur les sols dégradés. Ces variations montrent à l'instar des travaux antérieurs (Toutain et Piot, 1980 ; Grouzis, 1988 ; Kiema et al., 2001) que le travail du sol a des effets bénéfiques sur la structure et la composition floristique.

La valeur des pâturages dépend des espèces présentes dont l'intérêt fourrager détermine l'exploitation pastorale. Certaines espèces sont très appétées et d'autres moins appétées ou pas du tout. De toutes ces espèces, certaines sont particulièrement productives sur le pâturage en raison de leur densité et ont des qualités fourragères allant d'excellente à nulle. L'indice spécifique de qualité dans cette étude a été utilisé pour effectuer les classements des espèces. Ce classement permet de situer les espèces les unes par rapport aux autres. La valeur pastorale varie également en fonction des contributions spécifiques de la végétation. Dans les parcelles étudiées, les espèces peu productives c'est-à-dire celles ayant des contributions spécifiques inférieures à $1 \%$ (Daget et Poissonet, 1971), ont été plus nombreuses que celles productives.

L'évaluation économique effectuée à partir du fourrage exploitable démontre que les marges nettes de production s'élèvent à 15 150 FCFA sur les parcelles aménagées et 7 860 FCFA sur les témoins. Cette application tient compte également du coût du $\mathrm{kg}$ de fourrage durant les périodes critiques d'affourragement au Sahel qui est de 60 FCFA le kg (Bougouma et al., 2001) et des coûts de l'aménagement de la DL + SC à l'hectare qui est de 60000 FCFA (PSB, 2003). De ce fait, l'aménagement peut être considéré comme largement rentable.

L'analyse physico-chimique des sols des parcelles aménagées et leur témoin a montré des effets sensiblement faibles des traitements sur les variations de la composition chimique. D'un constat général, toutes les parcelles sont pauvres en azote et en phosphore, respectivement $0,036 \%$ et 75,8 à $76,9 \mathrm{mg} / \mathrm{kg}$ mais restent comparables aux sols des vieilles jachères de 30 ans et les jeunes défriches cultivées depuis 30 ans des régions soudaniennes de Saria, Gonsé et Korhogo $(0,06$ à 0,01$)$ rapporté par Roose (1981). Les pourcentages de carbone varient dans les mêmes fourchettes $(2,78$ à $2,63 \%)$ tandis que les $\mathrm{PHeau}$ et $\mathrm{PHCl}$ restent comparables. Cette évaluation montre que les sols dégradés qui ont été aménagés disposent de potentialités physico-chimiques suffisantes pour induire de bons niveaux de production fourragère.

Le bilan de la production annuelle des nutriments montre de fortes productions sur les parcelles aménagées en raison du bilan positif de la disponibilité en eau (bonne infiltration dans le sol travaillé) (Toutain et Piot, 1980 ; Grouzis, 1988 ; Hien, 1995 ; Kiema et al., 2001). Les productions d'azote et de phosphore ont été améliorées en dépit de l'absence de différences significatives entre les composants chimiques du sol et confirment que cette augmentation de la production est surtout le fait des changements de la structure du sol qui permet une meilleure économie de l'eau et la rétention des semences. Ces mesures démontrent que les aménagements permettent une forte mobilisation de nutriments dont une grande partie peut être mise à la disposition de la production animale. L'apparition de Cassia obtusifolia suggère cependant que des tests d'alimentation des animaux à base de cette espèce soient effectués pour permettre une meilleure valorisation des impacts des aménagements.

Par ailleurs, compte tenu du niveau assez limité de la disponibilité des nutriments dans le sol, la production suscitée par les aménagements aura à moyen terme un effet d'appauvrissement des sites aménagés si les exploitations sont maximales. L'enrichissement des sols aménagés en phosphore et NPK associé à un modèle d'exploitation devrait constituer une alternative pour contenir les impacts négatifs des niveaux d'exploitations actuelles. Des 
recherches doivent donc également être poursuivies pour montrer l'évolution de la fertilité des sols aménagés en demi-lune et soumis à une capacité de charge maximale.

\section{Conclusion}

Les travaux de récupération des pâturages dégradés par la technique de DL associée au SC ont montré les possibilités d'amélioration de la production fourragère. L'expérience menée dans les quatre villages révèle effectivement les possibilités d'augmentation de la production fourragère. Les compositions chimiques des terres nues ont révélé que leur qualité chimique n'était pas la principale raison du manque de production $\mathrm{du}$ fourrage mais plutôt les faiblesses en matière d'infiltration de l'eau dans le sol et le stockage de semences fourragères.

Il apparaît alors nécessaire que dans la lutte contre la dégradation des terres et pour l'amélioration des pâturages cette technique, au regard des effets positifs évidents enregistrés, soit mise à la disposition des communautés rurales. Cette technique est viable et d'intérêt stratégique car il est possible d'aménager de très grandes superficies, les travaux étant faits au tracteur. La planification, l'aménagement et la gestion des terroirs peuvent trouver en cette technique un outil favorable pour la recolonisation des grandes surfaces dégradées. Des investigations doivent cependant se poursuivre pour établir d'autres impacts (positifs et négatifs) de la technologie.

\section{REFERENCES}

Boudet G. 1991. Manuel sur les Pâturages Tropicaux et les Cultures Fourragères $\left(2^{\mathrm{e}}\right.$ édn). IEMVT: France.

Bougouma V, Nignan M, Kiema A. 2001. Etude de la Commercialisation des Fourrages au Burkina Faso. INERA/DPA: Ouagadougou.

Claude J, Grouzis M, Milleville P. 1991. Un Espace Sahélien; la Mare d'Oursi. ORSTOM: Burkina - Faso, France.

Daget P, Poissonet J. 1971. Une méthode d'analyse phytosociologie des prairies. Critères d'application. Ann. Agron., 22(1): 5-41.
Daget P, Poissonet J. 1972. Un procédé d'estimation de la valeur pastorale des pâturages. Revue Fourrages, 46: 31- 39.

De Wispelaere G. 1990. Dynamique de la désertification au Sahel du Burkina Faso. Cartographie de l'évolution et recherche méthodologique sur les applications de la télédétection. Mémoire pour le diplôme d'ingénieur, Cons. Arts et Métiers, Paris, p. 346.

Gaston A, Botte F. 1971. Étude Agrostologique de Tin Arkachen (République de Haute Volta). IEMVT, Maison Alfort: France.

Godron M, Daget P, Long G, Lefloch E, Poissonet J, Sauvage C, Wacquant JP. 1968. Code pour le Relevé Méthodique de la Végétation et du Milieu. Edition. CNRS: Paris.

Grouzis M. 1988. Structure, productivité et dynamique des systèmes écologiques sahéliens (Mare d'Oursi, Burkina Faso). Thèse de Doctorat d'État es sciences, Université de Paris Sud, Paris, p. 336.

Hien FG. 1995. La régénération de l'espace sylvo-pastoral au Sahel: Une étude de l'effet des mesures de conservation des eaux et des sols au Burkina Faso. PhD thesis, Université de Wageningen, Wageningen, p. 194.

Kaboré-Zoungrana CY. 1995. Composition chimique et valeur nutritive des herbacées et des ligneux des pâturages naturels soudaniens et des sous-produits du Burkina Faso. Thèse doctorat d'Etat es Sciences Naturelles, Université de Ouagadougou, Ouagadougou p. 224.

Kiema A. 1994. Étude des petits ruminants dans trois systèmes d'élevage traditionnel en zone soudano-sahélienne : paramètres zootechniques et utilisation des espaces pastoraux par le bétail. Mémoires d'ingénieur d'élevage, IDR/Université de Ouagadougou, Ouagadougou, p. 127.

Kiema A, Ouédraogo T, Nianogo AJ, Sanou S. 2001. Effets des cordons pierreux et du scarifiage sur la régénération des pâturages naturels en région sahélienne du Burkina Faso. Revue Science et Technique, Série Science Naturelle et Agronomie, 25(2): 99-113. 
Levang P. 1978. Biomasse Herbacée de Formations Sahéliennes. Etude Méthodologique et Application du Bassin Versant de la Mare d'Oursi. Lutte contre l'aridité dans l'Oudalan DGRST/ ORSTOM, ACC: France.

PSB. 2003. Rapport annuel d'activité 2002. Programme Sahel Burkinabé (PSB)/GZ, Burkina Faso.

Roose E. 1981. Dynamique actuelle des sols ferralitiques et ferrugineux tropicaux d'Afrique de l'Occidentale. Etude expérimentale des transferts hydrologiques et biologiques des matières sous végétations naturelles ou cultivées. Thèse Doc. Es Sciences, Univ. D’Orléans, Paris, p. 159.

Scheffe H. 1959. The Analysis of Variance. Wiley: New York.
Toutain B, De Wispelaere G. 1978. Etude et Cartographie des Pâturages de l'ORD du Sahel et de la Zone de Délestage au Nord-Est de Fada N'Gourma (Haute Volta) (Tome I); Les Pâturages Naturels et leur mise en Valeur (Tome II); Les Plantes, Ecologie, Noms Vernaculaires, Intérêt Fourrager (Tome III). Cartographie. Maison-Alfort, IEMVT: France.

Toutain B, Piot J. 1980. Mise en Défens et Possibilité de Régénération des Ressources Fourragères Sahéliennes. Etudes Expérimentales dans le Bassin Versant de la Mare d'Oursi. (Haute Volta). IEMVT : France. 